Abstracts: Session II

genes on a microarray. Thirty genes were upregulated and eighty downregulated by fourfold or more over background. To confirm subtraction efficiency, we picked approximately 700 clones from the C225-upregulated subtraction library; spotted their inserts, amplified by the polymerase chain reaction, on glass slides and hybridized them with the subtracted cDNAs. The majority of the clones exhibit high intensity when hybridized with the upregulated cDNAs and low intensity when hybridized with the downregulated cDNAs. The differential expression of these clones is now being confirmed by quantitative polymerase chain reaction with revserse transcription or northern blot analysis, and their function will be evaluated using cell differentiation and motility assays.

Onay, U. Venus [68]

\section{Identifying the role of single-nucleotide polymorphisms in breast cancer risk using microarray and mass spectrometry technologies}

\author{
U. Venus Onay ${ }^{1,2}$, A. Julia Knight ${ }^{2,3}$ \& Hilmi Ozcelik Hi, $^{1,4}$ \\ ${ }^{1}$ Department of Pathology and Laboratory Medicine, Mount Sinai Hospital, \\ Toronto, Ontario, Canada \\ ${ }^{2}$ Samuel Lunenfeld Research Institute, Mount Sinai Hospital, Toronto, Ontario, \\ Canada \\ ${ }^{3}$ Department of Public Health Sciences, University of Toronto, Toronto, Ontario, \\ Canada \\ ${ }^{4}$ Department of Pathobiology and Laboratory Medicine, University of Toronto, \\ Toronto, Ontario, Canada
}

A considerable proportion of the familial aggregation of breast cancer is probably due to alleles of low penetrance. Although their contribution to risk is relatively small, they can contribute to a large proportion of breast cancer cases in the population, because the risk-conferring alleles of these genes are common. The candidate gene approach is one of the most logical and practical strategies to identify these risk-enhancing, low-penetrant variants. A major obstacle in investigating the risk associated with multiple candidate genes has been the lack of large-scale genotyping technologies. Recently developed microarray and matrix-assisted laser desorption-ionization time-of-flight (MALDI-TOF) mass spectrometry technologies are ideally suited to the problem of high-throughput genotyping. We are relying on the power of these techniques to carry out a population-based breast cancer case-control study for 32 single-nucleotide polymorphisms (SNPs). We selected these SNPs from genes of crucial pathways that are frequently perturbed in cancers, such as those governing the cell cycle, carcinogen metabolism and the immune response pathways. We are currently developing both microarray and MALDI-TOF mass spectrometry techniques so that both can use the primer extension method to detect the allelic variants of SNPs. Both techniques are used in combination on a panel of cases involving several SNPs, to assess specificity and reproducibility. The combination of both methods will be a powerful tool for genotyping a large number of SNPs in a large number of cases. Advances in our knowledge of disease etiology will significantly expand our abilities to design strategies for the prevention of breast cancer development and the inhibition of its progression.
Orr, Michael

[69]

\section{Discovery of $\mathbf{8 3 0}$ candidate therapeutic targets and diagnostic markers for breast cancer using oligonucleotide microarray technology}

Michael Orr, Amanda Williams, Lisa Vogt, Joseph Boland, Harry Yang, Jeffrey Cossman \& Uwe Scherf

Gene Logic Inc., 708 Quince Orchard Road, Gaithersburg, Maryland 20878, USA

To identify differentially expressed genes in infiltrating ductal carcinoma (IDC) of the breast, we measured the gene expression profiles of 15 IDC and 13 normal human breast tissues, using the Affymetrix GeneChip array platform for simultaneous analysis of over 60,000 genes. Fold-change comparison between normal and IDC breast tissue samples revealed 830 genes that were statistically over- or underexpressed by threefold or greater in the IDC samples. We identified 286 overexpressed genes and 544 underexpressed genes. Furthermore, the 830 genes were evaluated for tissue-specific expression by E-northern analysis of 28 different normal tissues, revealing tissue-specific candidate targets. We performed further analysis utilizing principal component analysis or hierarchical clustering with 5,467 prefiltered genes to determine if gene expression profiles could be used to distinguish between IDC and normal breast tissue samples. Both PCA and HCA indicated two distinct populations for the normal and tumor-derived samples, except for two samples, based on gene expression patterns. One aberrant tumor sample was explained by its histology, which showed only partial $(<20 \%)$ involvement by malignant cells. We also employed cluster analysis to evaluate differences in samples at the single-gene level. Examination of a particular cluster of overexpressed genes displayed a multitude of differentially expressed sequence tags along with genes for topoisomerase II, cyclin B, CDC2, KI-67, and thymidine kinase, suggesting some functional similarities. Our studies provide a rational basis for additional analysis of differentially expressed genes in breast cancer, which may lead to the identification of therapeutic targets and diagnostic markers.

Parodi, Silvio

[70]

\section{Innovative leads for antineoplastic drugs suggested by a more intimate familiarity with structural motifs of oncoproteins}

Maria Pia Pescarolo ${ }^{1,2}$, Luca Bagnasco ${ }^{1,2}$, Davide Malacarne ${ }^{1}$, Antonella Melchiori ${ }^{1}$, Piera Valente ${ }^{1,2}$, Enrico Millo ${ }^{3}$,

Silvia Bruno ${ }^{4}$, Stefania Basso ${ }^{5} \&$ Silvio Parodi ${ }^{1,2}$

${ }^{1}$ Laboratory of Experimental Oncology, IST Genoa, Genoa, Italy

${ }^{2}$ Department of Oncology, Biology and Genetics, University of Genoa, Genoa, Italy

${ }^{3}$ Department of Experimental Medicine (Biochemistry), University of Genoa, Italy, Genoa, Italy

${ }^{4}$ Department of Experimental Medicine (Human Anatomy), University of Genoa, Genoa, Italy

${ }^{5}$ Immmunopharmacology Section, IST/CBA, Genoa, Italy

Proteins, although structurally unsuitable for their own replication, are the structures that translate an abstract DNA sequence into real action, making possible catalysis, signaling and supramolecular morphologies. Protein targets have most often been recognized by starting from antineoplastic effects initially observed at the level of an organism or a cell and working in a reductionist manner. However, no oncoproteins have been identified as cancer chemotherapy targets through this 